\title{
Segmentation of Neck Lymph Nodes in CT Datasets with Stable 3D Mass-Spring Models
}

\author{
Jana Dornheim ${ }^{1}$, Heiko Seim ${ }^{1}$, Bernhard Preim ${ }^{1}$, Ilka Hertel $^{2}$, \\ and Gero Strauss ${ }^{2}$ \\ ${ }^{1}$ Otto-von-Guericke-Universität Magdeburg, Germany \\ ${ }^{2}$ Hals-Nasen-Ohren-Universitätsklinik Leipzig, Germany
}

\begin{abstract}
The quantitative assessment of neck lymph nodes in the context of malign tumors requires an efficient segmentation technique for lymph nodes in tomographic 3D datasets. We present a Stable 3D MassSpring Model for lymph node segmentation in CT datasets. Our model for the first time represents concurrently the characteristic gray value range, directed contour information as well as shape knowledge, which leads to a much more robust and efficient segmentation process. Our model design and segmentation accuracy are both evaluated with lymph nodes from clinical CT neck datasets.
\end{abstract}

\section{Motivation}

The assessment of lymph nodes plays an important role in the diagnosis, staging, treatment and therapy control of malign tumors and their metastases. MRI and CT scans of the respective regions allow for a 3D assessment of the pathological situation, however, an exact quantitative analysis demands an efficient segmentation of the lymph nodes in the 3D datasets. Lymph node segmentation currently has to be performed manually, which is unfeasible in the time-critical clinical routine. We present an efficient model-based segmentation technique for enlarged lymph nodes in CT datasets of the neck. Automatic segmentation of neck lymph nodes is difficult due to the following problems:

- Neck lymph nodes often touch or infiltrate adjacent soft tissue (Fig. 1 (b), (c)), which lets all purely gray value-based segmentation techiques fail.

- Adjacent high-contrast structures often lead to high gradient magnitudes in the local neighbourhood (Fig. 1] (d)), which may distract model-based segmentation approaches based on contour strength.

- The lymph nodes' interior homogeneity may be disturbed by a central necrosis (Fig. 1 (e)).

- The size of metastatic lymph nodes varies significantly.

\section{State of the Art}

The segmentation of lymph nodes in CT image data is long been known as an open problem. Rogowska et al. [1] evaluated elementary segmentation techniques 


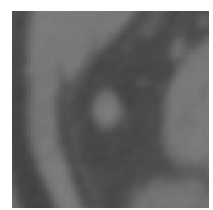

(a)

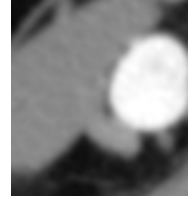

(b)

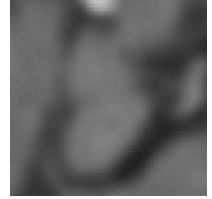

(c)

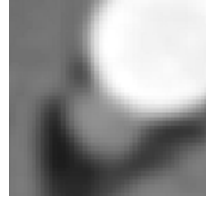

(d)

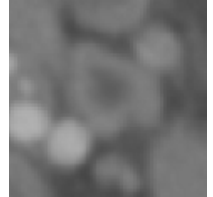

(e)

Fig. 1. Different anatomical situations in CT head and neck data: (a) isolated neck lymph node, (b) neck lymph node adjacent to M. sternocleidomastoideus, (c) two adjacent lymph nodes, (d) lymph node touching high-contrast structure (blood vessel), (e) lymph node with a central necrosis (dark).

(e.g. Thresholding, Watershed Transformation, ...) for lymph node segmentation, and conclude that a high degree of model knowledge is needed for a reliable segmentation. Honea et al. 2] used a slicewise Active Contour as a first modelbased segmentation approach. They extended their approach by using a 3D Active Contour model [3]. Both their 2D and 3D approach are purely based on contour information (gradient magnitude) and shape knowledge (represented by a viscosity condition). Yan et al. 4] exploited the characteristic gray value range as well as the contour information of lymph nodes by using an improved 2D Fast Marching Method with an intensity-weighted speed term. However, no shape knowledge was used by their approach. Boundary leaking could only be avoided by a hard stopping criterion (bounding circle around the lymph node). All discussed approaches require high interaction effort. No quantitative evaluation on clinical CT datasets was performed. No approach integrates all, contour information, characteristic gray value range and shape knowledge.

Besides lymph node segmentation techniques, newer approaches are known on the segmentation of small homogeneous soft tissue structures in CT data (e.g. pulmonary nodules [5]). However, these approaches presume a homogeneous background, which makes them inappropriate for the segmentation of neck lymph nodes in their complex anatomical environment.

\section{Method}

We employ Stable 3D Mass-Spring Models (SMSMs) introduced by Dornheim et. al. [6] for the neck lymph nodes segmentation, which allow for explicit modelling of shape and appearance, while leaving the modelled object size flexible.

\subsection{Stable 3D Mass-Spring Models}

Mass-Spring Models (MSMs) are physically-based models known from soft tissue simulation. They use spring forces to describe object shape by the distance of connected mass points. MSMs can also be applied as deformable models for segmentation. In this case, no simulation of a real physical situation is required. Instead, the model dynamics is used to adapt a deformable shape to image 
information by a local optimization process. For this purpose however, the representation of shape by spring forces alone is not stable enough and often leads to model shape collapse, especially in $3 \mathrm{D}$.

SMSMs extend conventional MSMs by using not only the springs' rest lengths but also their rest directions to describe the model. Whenever a spring deviates from its rest direction, a torsion force is created to restore its original direction. Thus, the object shape is mainly represented by the springs' rest directions, while their rest lengths represent the object's scaling.

The external model forces of an SMSM are created by sensors at the mass points. They transform the image information at their current position into a sensor force acting on the associated mass point. For the segmentation of lymph nodes, two types of sensors are of interest: intensity sensors, dragging their associated mass to voxels of a given gray value range, and direction-weighted gradient sensors, steering towards voxels at gradients of high magnitude and preferable expected direction.

\subsection{Design of the Lymph Node Model}

The manual segmentation of lymph nodes exploits a set of characteristic features, which all together allow the human observer to detect lymph nodes in CT datasets. The following features were confirmed by our clinical partner:

(i) Ellipsoid to spherical shape with a varying ratio of longitudinal and transversal radii. This ratio is approximately $2: 1$ for healthy lymph nodes. With metastatic lymph nodes, it approaches 1:1.

(ii) Medium strength of contour information for isolated lymph nodes. Contours of non-isolated lymph nodes can be interrupted by adjacent soft tissue or amplified by adjacent high-contrast structures.

(iii) Homogeneous gray value (soft tissue) in the lymph node interior (with the exception of lymph nodes with a central necrosis).

(iv) Size varies strongly: neck lymph nodes are clinically relevant at a size of $1 \mathrm{~cm}$, the maximum size can be assumed as $3 \mathrm{~cm}$, since larger lymph nodes must be considered "real" metastases, which are often not spherical.

To model the ellipsoid to spherical shape of the lymph nodes (i), a 3D SMSM was created as a polygonic sphere surface (Fig. 2 (a)). At each mass point, a direction-weighted gradient sensor was placed, in order to model the contour information (ii) including the local contour normal direction we expect to find here.

To incorporate the additional knowledge about the inner gray value of the lymph nodes (iii) into the model, we created a second, inner sphere surface (Fig. 2 (b)) as a downscaled copy (scaling factor 0.9) of the first surface. At each mass point of this inner surface, an intensity sensor was placed, searching for the specific gray value of the target lymph node. We chose to arrange these inner sensors as another surface, instead of distributing them evenly across the lymph node interior, in order to segment lymphnodes with inner necrosis. 


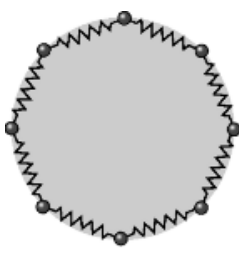

(a)

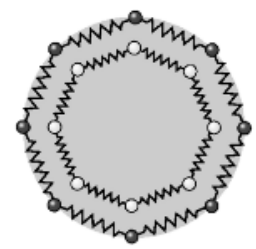

(b)

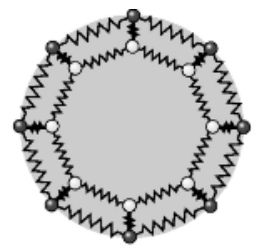

(c)

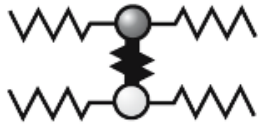

(d)

Fig. 2. Stages of the model construction (schematic 2D view): (a) First surface model with gradient sensors (dark gray). (b) An inner front of intensity sensors (white) is added. (c) Both fronts are connected by stiff springs, with the effect, that the associated gradient and intensity sensor act as a functional unit (d).

In a final modelling step, each gradient sensor of the outer surface was connected to its associated intensity sensor at the inner surface (Fig. 22(c)). To make both associated sensors work as a functional unit, we assigned all springs between the two surfaces a very high stiffness (factor 1000 to all other springs) (Fig. 2 (d)). This way, each intensity sensor keeps its associated gradient sensor close to the homogeneous lymph node interior and prevents model distraction by false strong gradients (ii). All other springs, i.e. the springs belonging to one of the surface submodels, were assigned a very low stiffness, in order to represent the unknown size of the lymph nodes (iv). In connection with the shape-preserving torsion forces, model shape stability is guaranteed [6] and can be expected to bridge gaps in the lymph node contour (ii) by shape knowledge.

\subsection{Segmentation Process}

The segmentation process consists of the following three steps:

Step 1: Initial placement of the model in the dataset. Since the model simulation is based on a local optimization process [6], the model should initially be placed inside the target lymph node. Thereto, the user specifies a seed point, at which a scaled model of minimal size $(0.3 \mathrm{~mm})$ is placed automatically, assuming that all lymph nodes are at least twice as big as this minimal size. Alternatively, the initial model can be specified by two seed points at the lymph node contour, whose distance is used as the intial model's diameter. This more precise initialization leads to faster model convergence. After model initialization, the rest lengths of all springs are set to their current lengths, so that the new model size is its default size.

Step 2: Determination of gray value range. The expected gray value range of the target lymph node is estimated from the given start position. Thereto, the gray value range of all pixels on the initial sphere diameter is used. The model's intensity sensors are then programmed to react only to this gray value range.

Step 3: Model adaptation. Finally, the model adaptation is run (Fig. 3) with the runtime parameters shown in Table1, They were adjusted once by experimental 


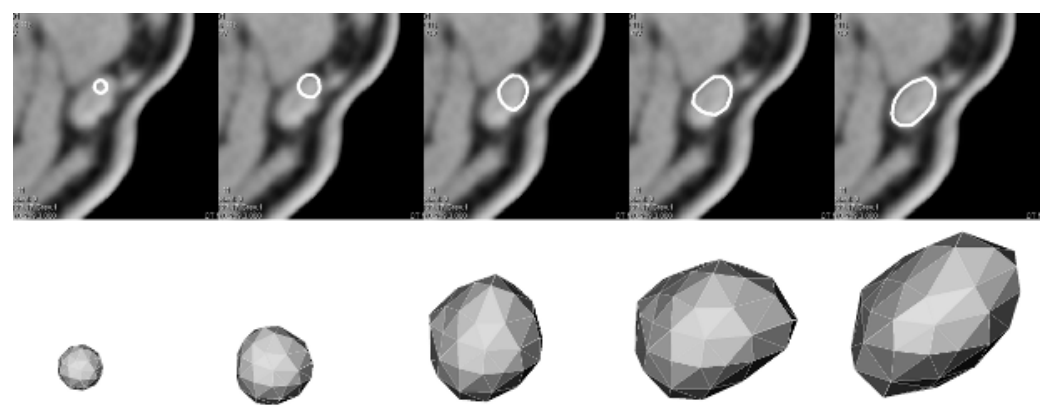

Fig. 3. $2 \mathrm{D}$ and $3 \mathrm{D}$ view of the model adaptation process

model runs and then used for all datasets during the evaluation phase. The model adaptation is automatically stopped, when every mass point remains inside a certain $\varepsilon$ environment for $n$ simulation steps. Due to the use of a damping force, the model converges and stops.

\section{Results and Evaluation}

$11 \mathrm{CT}$ datasets of the neck region with 146 enlarged lymph nodes were available for the evaluation. The datasets stem from different CT devices, the quality of the datasets varied significantly with respect to signal-to-noise ratio, horizontal resolution and slice distance. For 40 lymph nodes in 5 of the 11 datasets, a gold standard (segmentation by a radiologist) was given, as well as manual segmentations by two experienced users. All evaluation was performed with the created SMSM (100 masses, 338 springs), without manual correction of the results.

\subsection{Qualitative Evaluation}

We first performed a qualitative study to validate the model design and to reveal, which components of the model are essential. Thereto, we started the model simulation with different model components disabled on specific lymph nodes of the 11 test datasets. The following results could be verified.

Table 1. Model simulation parameters (according to [6])

\begin{tabular}{c|c|c|c}
\hline Parameter Category & Parameter Description & Symbol & Parameter Value \\
\hline \hline \multirow{3}{*}{ Model adaptation } & Simulation step size & $\triangle t$ & 0.02 \\
& Tolerance (stopping criterion) & $\varepsilon$ & $0.01 \mathrm{~mm}$ \\
& Simulation steps (stopping criterion) & $\mathrm{n}$ & 5 \\
\hline \multirow{3}{*}{ Weighting } & Torsion forces & $w_{t}$ & 25 \\
of force components & Spring forces & $w_{f}$ & 20 \\
& Gradient sensor forces & $w_{s_{g}}$ & 9 \\
& Intensity sensor forces & $w_{s_{i}}$ & 6 \\
\hline
\end{tabular}




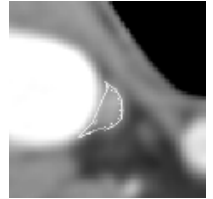

(a)

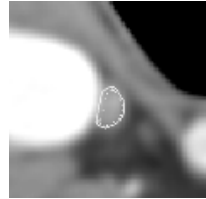

(b)

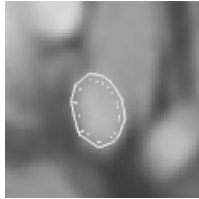

(c)

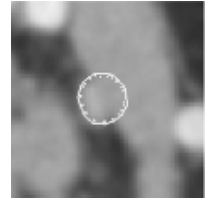

(d)

Fig. 4. Effect of the torsion forces on the segmentation results: (a) distraction of gradient sensors by stronger neighbouring gradients. In (b), this distraction is prevented by the shape-stabilizing effect of the torsion forces. In (c) and (d), the shape knowledge of the model extrapolates missing contour information.

Shape representation by torsion forces. The torsion forces proved to be suitable for the representation of the shape knowledge. We first tested this behaviour with lymph nodes touching high-contrast structures. The shape stability gained by the use of torsion forces prevents the model from being distracted by the strong gradients of these neighbouring structures (Fig. 4 (a) and (b)). In cases with lymph nodes adjacent to soft tissue structures, the torsion forces caused the model to extrapolate the organic lymph node surface by shape knowledge at places with weak or missing contour information (Fig. 4 (c) and (d)).

Adaptation to lymph node size. Our model proved to be able to adapt to lymph nodes of different sizes even for imprecise initial positioning. We verified this behaviour with 8 isolated lymph nodes and a very small model initialization. In all cases, the model adapted completely to the size of the target lymph node (Figure 3). Without torsion forces, the model collapsed in 4 of 8 cases.

Functional unit of gradient and intensity sensors. The combination of intensity and gradient sensors turned out to be essential for the successful segmentation. We verified this with 10 lymph nodes adjacent to high-contrast structures. Without intensity sensors, the strong neighbouring gradients often attracted single gradient sensors, which lead to "leaking" into the high-contrast structure (Fig. 5] (a)). In cases of small lymph nodes, often the whole model adapted to the complete shape of the blood vessel (Fig. 5(c)). With the combined use of gradient and intensity sensors, this behaviour was completely eliminated.

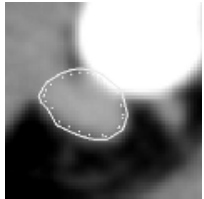

(a)

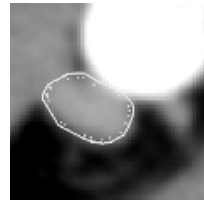

(b)

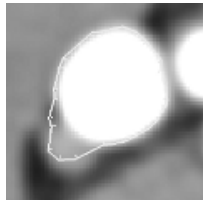

(c)

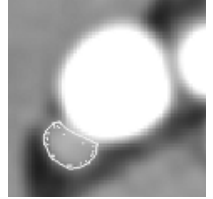

(d)

Fig. 5. Segmentation results with gradient sensors only (a, c), and with combined gradient and intensity sensors $(b, d)$. 
The results of our qualitative analysis show, that only the combination of all above-described model components - shape knowledge, gray value and gradient information - enables a robust lymph node segmentation.

\subsection{Quantitative Evaluation}

To evaluate the accuracy of our model-based segmentation, we assembeled a quantitative analysis based on the 40 lymph nodes for which a gold standard was available, which was the case for all problem classes of homogeneous lymph nodes (see Fig. 1 (a)-(d)). These 40 lymph nodes were equally distributed across 5 of the $11 \mathrm{CT}$ neck datasets.

We used the 2-point initialization to place the model in each of the 40 lymph nodes and started the model simulation with the parameters given in Table 1. The model simulation stopped automatically for all 40 lymph nodes after 2 to 30 seconds on a modern standard PC (3.2 GHz Pentium 4). The model's segmentation result, as well as the manual expert segmentations for all 40 lymph nodes were then compared to the given gold standard (see Fig. 6] and Table 2 for results). Our analysis showed, that the accuracy of our segmentation model is comparable with the accuracy of the given manual segmentations.

The mean surface distance of the model's segmentation results varied between $0.3 \mathrm{~mm}$ and $0.6 \mathrm{~mm}$, while the Hausdorff distance varied between $1.7 \mathrm{~mm}$ and a maximum of $3.9 \mathrm{~mm}$. For the Hausdorff distance, the biggest errors stem from different estimation of the upper and lower border of the lymph node, which is directly correlated with the slice distance (up to $3.9 \mathrm{~mm}$ ). Since this deviation could also be observed for manual segmentations, it shows that this is still a general problem, which can only be reduced by smaller slice distances.

\section{Conclusion}

We presented a new 3D segmentation technique for lymph nodes in CT datasets, using deformable models (SMSMs). This allowed for the first time to incorporate the three characteristic features of lymph nodes - gray value range, contour information and shape knowledge - into one single 3D model. This results in a robust and efficient segmentation process with minimal interaction needed.

Our method was evaluated with 40 lymph nodes from clinical CT datasets and could be proved to be comparable to the manual segmentation results of two experienced users.
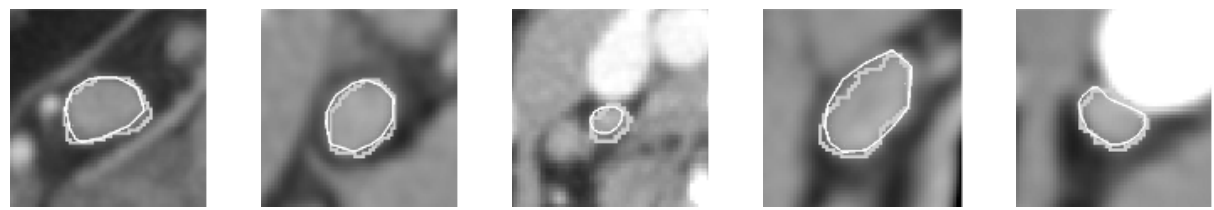

Fig. 6. Segmentation results of the lymph node model (white) and gold standard segmentation (light gray) 
Table 2. Results of the quantitative Evaluation

\begin{tabular}{l|l|l|l|l|l|l}
\hline & Dataset & 1 & 2 & 3 & 4 & 5 \\
\hline \hline \multirow{3}{*}{$\begin{array}{l}\text { Mean Surface Distance } \\
(\mathrm{mm})\end{array}$} & User A & 0.420 & 0.353 & 0.401 & 0.311 & 0.296 \\
& User B & 0.488 & 0.406 & 0.444 & 0.271 & 0.279 \\
& Model & 0.421 & 0.309 & 0.596 & 0.583 & 0.416 \\
\hline \multirow{3}{*}{ Hausdorff Distance } & User A & 3.513 & 2.401 & 3.238 & 1.213 & 1.575 \\
$(\mathrm{~mm})$ & User B & 4.113 & 2.644 & 3.225 & 1.150 & 1.725 \\
& Model & 3.913 & 2.350 & 3.825 & 1.700 & 1.925 \\
\hline \multirow{2}{*}{ Volumetric Segmentation } & User A & 45.88 & 69.38 & 43.25 & 35.13 & 37.00 \\
Error (\%) & User B & 45.63 & 65.25 & 50.88 & 29.88 & 33.13 \\
& Model & 44.38 & 50.75 & 51.38 & 51.75 & 38.88 \\
\hline
\end{tabular}

\section{References}

1. Rogowska, J., Batchelder, K., Gazelle, G., Halpern, E., Connor, W., Wolf, G.: Evaluation of selected two-dimensional segmentation techniques for computed tomography quantification of lymph nodes. In: Investigative Radiology. Volume 13. (1996) $138-145$

2. Honea, D.M., Ge, Y., Snyder, W.E., Hemler, P.F., Vining, D.J.: Lymph node segmentation using active contours. In: Proc. SPIE Medical Imaging 1997: Image Processing. Volume 3034. (1997) 265-273

3. Honea, D., Snyder, W.E.: Three-dimensional active surface approach to lymph node segmentation. In: Proc. SPIE Medical Imaging 1999: Image Processing. Volume 3361. (1999) 1003-1011

4. Yan, J., ge Zhuang, T., Zhao, B., Schwartz, L.H.: Lymph node segmentation from CT images using fast marching method. Computerized Medical Imaging and Graphics 28 (2004) 33-38

5. Kuhnigk, J.M., Dicken, V., Bornemann, L., Wormanns, D., Krass, S., Peitgen, H.O.: Fast automated segmentation and reproducible volumetry of pulmonary metastases in CT-scans for therapy monitoring. In: Medical Image Computing and ComputerAssisted Intervention - MICCAI 2004. Volume 3217. (2004) 933-941

6. Dornheim, L., Tönnies, K., Dornheim, J.: Stable dynamic 3d shape models. In: International Conference on Image Processing (ICIP). (2005) 